\title{
Propuesta de una herramienta para la medición y evaluación en el desarrollo de nuevos productos
}

\author{
Proposal of a tool for the measurement and \\ evaluation in the development of new products
}

\author{
Laura Marcela Gaviria-Yepes ${ }^{1 *} \quad$ Alejandro Valencia-Arias ${ }^{1}$ \\ Recibido 26 de septiembre de 2018, aceptado 17 de junio de 2019 \\ Received: September 26, 2018 Accepted: June 17, 2019
}

\begin{abstract}
RESUMEN
Se realizó un estudio cuyo objetivo fue proponer una herramienta para medir y evaluar el proceso de desarrollo de nuevos productos, basado en fundamentos teóricos como State-Gate y Balanced Scorecard. Para su construcción, se siguieron 4 fases, la primera consistió en una revisión de conceptos claves, la segunda se desarrolló a través de un estudio bibliométrico, la tercera se centró en un análisis del contexto empresarial y la cuarta en el desarrollo de la herramienta de medición, constituyendo la contribución teórica de la investigación. Así, con la unión de ambas herramientas, se creó una adaptación de Balanced Scorecard para el proceso de desarrollo de nuevos productos, como una propuesta para seguir este tema, y se establecieron objetivos estratégicos para cada etapa, acompañados de indicadores que permitirán medir el estado de cada objetivo y en caso de no cumplirse, no será posible avanzar hacia la siguiente etapa; esta herramienta también planteó cinco puertas con sus respectivas actividades: definición de ideas, construcción del enfoque, desarrollo, prueba y validación; y finalmente lanzar y publicar la revisión de la versión. Con esto se concluye que el desarrollo de nuevos productos acompañados por la gestión de la innovación es fundamental para que las empresas crezcan y generen rentabilidad, también para promover el empleo y promover la competitividad del país, promoviendo el desarrollo económico; esto es posible cuando hay herramientas de planeación estratégica disponibles que permiten llevar a cabo las funciones de planeación, organización, dirección y control de manera efectiva.
\end{abstract}

Palabras clave: Balanced Scorecard, State-Gate, competitividad, gestión de la innovación, planeación estratégica.

\section{ABSTRACT}

A study was carried out whose objective was to propose a tool to measure and evaluate the development process of new products, based on theoretical foundations such as State-Gate and Balanced Scorecard. For its construction, 4 phases were followed, the first consisted of a review of key concepts, and the second was developed through a bibliometric study, the third focused on an analysis of the business context and the fourth on the development of the tool measurement, constituting the theoretical contribution of the investigation. Thus, with the union of both tools, the Balanced Scorecard was created for the process of developing new products, as a proposal to follow this important issue, and strategic objectives were established for each stage, accompanied by indicators that will allow measuring the status of each objective and if not met, it will not be possible to advance to the next stage; this tool also raised five

\footnotetext{
1 Instituto Tecnológico Metropolitano. Facultad de Ciencias Económicas y Administrativas. Medellín, Colombia. E-mail: lauragaviria@itm.edu.co; jhoanyvalencia@itm.edu.co

* Autor de correspondencia: lauragaviria@itm.edu.co
} 
doors with their respective activities: definition of ideas, construction of focus, development, testing and validation; and finally launch and publish the revision of the version. This concludes that the development of new products accompanied by the management of innovation is essential for companies to grow and generate profitability, also to promote employment and promote the country's competitiveness, promoting economic development; This is possible when there are strategic planning tools available that allow to carry out the functions of planning, organization, direction and control in an effective way.

Keywords: Balanced Scorecard, State-Gate, competitiveness, innovation management, strategic planning.

\section{INTRODUCCIÓN}

Para que las empresas puedan mantenerse vigentes en las preferencias de los clientes, es fundamental que desarrollen nuevos productos y que estos a su vez, tengan un componente innovador [1], sin embargo, este proceso no es nada fácil ya que implica riesgos en tiempo, dinero y sobre todo, la posibilidad que finalmente el producto no resulte exitoso [2]. Para reducir la incertidumbre y mejorar la eficiencia, existen diferentes modelos para el desarrollo de nuevos productos, uno de ellos es el State-Gate System de Cooper [3], el cual se compone de etapas, las cuales se aprueban o no por medio del paso por puertas, lo que hace que el proceso sea mucho más controlado; sin embargo, también es importante hacer mediciones en cada etapa o desglosar por objetivos que sean fácilmente medibles. Así mismo, existe una herramienta de gestión muy popular en la planeación estratégica y se conoce como Balanced Scorecard [4], la cual monitorea aspectos de la estrategia organizacional.

Por otra parte, la planeación estratégica permite generar planes de trabajo con el fin de encaminarse hacia el cumplimiento de los objetivos organizacionales, en ese sentido, el desarrollo de nuevos productos es proceso que debe planearse y evaluarse de una manera adecuada con el fin de obtener resultados satisfactorios para la organización, es decir, reducir la incertidumbre y asegurar el logro de los objetivos, esto sobretodo en empresas de manufactura, las cuales deben generar productos tangibles.

El presente artículo propone una herramienta para medir y evaluar el proceso de desarrollo de nuevos productos, además de estimar los recursos, a partir de herramientas como el State-Gate y el Balanced Scorecard enfocado a empresas manufactureras, esta herramienta se ha logrado construir a partir de fundamentación teórica y práctica analizada en fuentes formales e informales con las cuales se realizó un análisis para crear una herramienta estándar, fácilmente adaptable y modificable para empresas de manufactura que la requieran para sus procesos.

\section{MARCO TEÓRICO}

\section{El proceso de desarrollo de nuevos productos}

Las tasas de fracaso en el desarrollo de nuevos productos son altas [5], mientras que unos procesos son cancelados sin haberse terminado, otros que logran salir, luchan por mantenerse a flote y en muchos casos no se logra un retorno económico [6] Para que este proceso sea exitoso, debe cumplir con tres objetivos de manera simultánea: el primero es maximizar el ajuste con los requerimientos del cliente, es decir, ofrecer productos solo ajustados a sus necesidades y no con características adicionales; el segundo es minimizar el tiempo del proceso de desarrollo, lo que puede generar ventajas como diferenciación y lealtad de marca, y por último, controlar los costos de desarrollo, manejando la eficiencia para garantizar retorno económico [6].

Por ello, en la medida que ha pasado el tiempo han surgido herramientas que contribuyen al proceso de desarrollo de nuevos productos, entre ellas se encuentran los procesos de etapa -puerta (State-Gate por su acepción en inglés), matriz de despliegue de calidad, diseño para la fabricación y diseño asistido por ordenador y fabricación asistida por ordenador [7]. Para efectos del estudio realizado, se optó por profundizar en la herramienta State-Gate, que se detalla a continuación:

\section{State-Gate: Modelo de puertas para la innovación en el desarrollo de nuevos productos}

Para desarrollar una innovación en un producto, se requiere realizar una investigación exhaustiva para obtener un buen resultado en el proceso, para 
esto, el modelo State-Gate es una buena opción por el alto grado de confiabilidad que representa [6].

El modelo State-Gate o modelo de Etapa - Puerta, fue diseñado por Robert Cooper a principios de los años 90 basado en estudios previos de Booz, Allen y Hamilton que datan de 1968 y significa Stage (seis etapas) Gate (cinco controles), es decir, cinco controles para seis etapas. Este modelo va desde lo conceptual hasta lo operacional y beneficia el proceso de desarrollo de nuevos productos [8]. Además, este es adaptable y permite su redimensionamiento a diferentes proyectos con niveles de riesgo distintos: desde desarrollos complejos y muy riesgosos, hasta extensiones y modificaciones de bajo riesgo. Igualmente, incorpora mejores prácticas de toma de decisiones incluso tarjetas de tanteo, criterios de éxito, mejor manejo de puertas y la orientación hacia la innovación abierta [9].

Así mismo, vale destacar que el enfoque del modelo de State-Gate desglosa el proceso de desarrollo de nuevos productos en cinco etapas o fases, las cuales están precedidas por una puerta de entrada o punto de decisión; donde las puertas cumplen una función de control de calidad para que nada sea dejado al azar. Éstas junto con sus tareas pueden ser observadas en la Figura 1.

Las puertas con sus respectivas etapas se definen de la siguiente manera: la primera etapa denominada medición del alcance, explora las necesidades del consumidor y si las oportunidades encajan en la realidad, además se realiza una evaluación de costos; en la segunda, conocida como construcción del caso de negocio, se realizan rigurosos estudios de factibilidad y se afinan los análisis de costos; para la tercera que es el desarrollo, se realizan procesos y ensayos de escala o prototipo, además de revisar la facilidad de construcción y operación del proceso; en la cuarta, que consiste en la evaluación y validación, se realiza la manufactura del producto y su valoración, que se constituye en un factor fundamental y crítico, así como la operabilidad y control del proceso para su escalamiento y optimización; en la última etapa que es el lanzamiento, se procede con la introducción del producto al mercado y se evalúan aspectos como: canales de distribución y mercadeo, facilidad de operación, control de proceso y evaluación prospectiva del mercado al producto [9].

Fuente: Elaboración propia a partir de (Schilling \& Martínez, 2008).
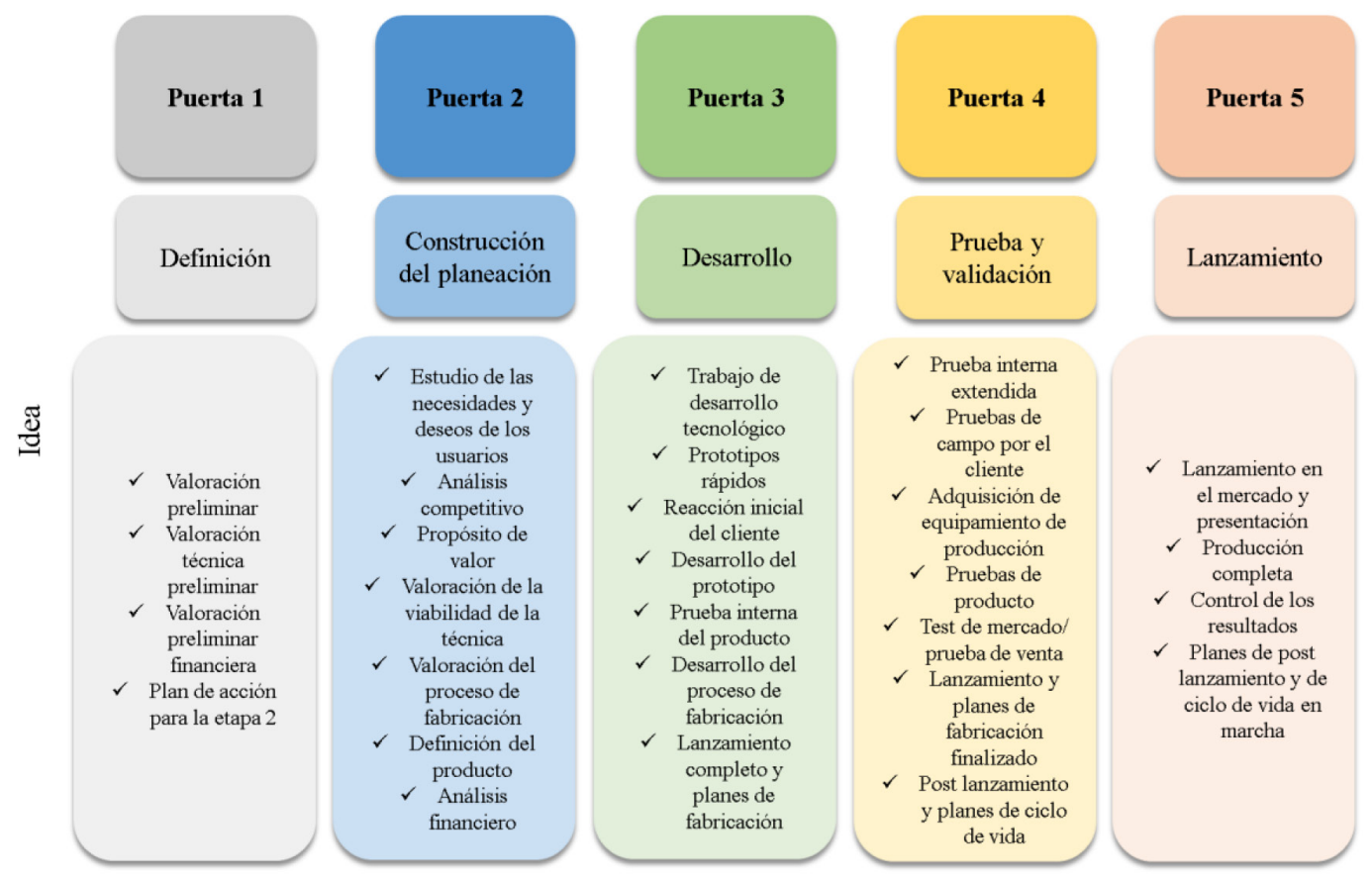

Figura 1. Proceso estándar de Stage - Gate, de la idea al lanzamiento. 


\section{El Balanced Scorecard (BSC) como herramienta de medición y gestión}

El Balanced Scorecard es también conocido como cuadro de mando integral. Es una herramienta de gestión que monitorea todos los elementos importantes en la estrategia de una empresa, desde la mejora continua y las alianzas hasta el trabajo en equipo [10]. Esta fue creada con el fin de obtener una visión integral de la organización, debido a que en tiempos anteriores solo se medía el desempeño a partir de indicadores financieros, y aunque estos son muy importantes, es fundamental monitorear también otros aspectos tal como los activos intangibles y las capacidades de producción [4].

Además, cabe resaltar que su utilidad no depende del tipo de empresa, sino de los problemas a los que se enfrenta [11], es decir, este se puede usar en todo tipo de empresas, desde las más pequeñas hasta las más grandes, todo dependerá de sus necesidades en el mediano y largo plazo, así como también el grado de definición de metas, objetivos, misión y visión [12]. En este sentido, el cuadro de mando integral ofrece una visión integral de la organización desde cuatro perspectivas, a continuación, en la Figura 2, se representa de manera gráfica cada una de ellas y la interacción entre las perspectivas:

De acuerdo con la anterior figura, es posible apreciar que el BSC se compone por cuatro perspectivas,

Fuente: Elaboración propia a partir de Dávila [13].



Figura 2. Perspectivas del Balanced Scorecard (BSC). siendo la primera de ellas la financiera, considerada el "corazón de la empresa" [14], en ella se analiza cómo se ve la organización ante los ojos de los inversionistas y si se están alcanzando las estrategias que contribuyen a la generación de valor económico y riqueza de éstos, siendo esta perspectiva la única que se refiere a activos intangibles; la segunda, llamada clientes, detalla cómo estos ven a la organización, si se encuentran satisfechos y si la compañía está enfocando todos los esfuerzos a sus segmentos de mercado; la tercera, denominada procesos internos y de negocio, evidencia el plan de la alta gerencia para crear valor económico de los clientes, aumentar el valor de la empresa y se identifican además, procesos críticos para establecer indicadores de gestión que permitan visualizar su desempeño; la cuarta y última perspectiva, innovación y aprendizaje, se enfoca en identificar recursos clave para innovar y mejorar, para esto se analizan tres niveles de contribución de las personas dentro de la organización: como individuo, como miembro de un equipo de trabajo y como alguien integrado a la cultura organizacional [15-16].

\section{METODOLOGÍA}

Se llevó a cabo un estudio cuyo objetivo consistió en proponer una herramienta para medir y evaluar el proceso de desarrollo de nuevos productos, a partir de fundamentos teóricos como el State-Gate y el Balanced Scorecard; con la finalidad de proveer un insumo que sea de utilidad para la gestión comercial de las organizaciones.

Así, se efectuó un rastreo en diferentes fuentes de la literatura científica y académica, donde se muestran aspectos relacionados con el proceso de desarrollo de nuevos productos, State-Gate System y el Balanced Scorecard; que permitió a los autores tener un panorama claro respecto a la fundamentación teórica. Además, de forma complementaria, se emplearon otras estrategias que le imprimieron rigor metodológico al estudio, tales como el análisis bibliométrico, la vigilancia tecnológica y comercial.

En este sentido, para dar cumplimiento al objetivo del estudio se realizaron actividades de vigilancia tecnológica, estudio bibliométrico y vigilancia comercial, las cuales brindaron elementos importantes para la elaboración de la herramienta. De este modo, 
el proceso de definición de la misma tuvo en cuenta un plan de cuatro fases, el cual puede apreciarse en la Figura 3.

Durante la primera fase, denominada revisión de conceptos teóricos, se establecieron las bases para el entendimiento y la conceptualización de los elementos clave, estos son las herramientas BSC y State-Gate; en esta fase se definieron las herramientas que tuvieran mayor afinidad al objetivo de investigación, para lograr esto, se consultaron fuentes humanas como lo son profesores e investigadores cercanos con el fin de obtener de primera mano los conocimientos necesarios para abordar herramientas que fueran pertinentes con el concepto a tratar, el cual es el desarrollo de nuevos productos, también se consultaron fuentes académicas como lo son las bases de datos, esto con el fin de lograr una mayor comprensión y articulación del tema. En la segunda, se llevó a cabo un análisis bibliométrico, en el cual se identificaron avances, tendencias y autores más representativos en los temas que intervienen en el desarrollo de la herramienta. El análisis bibliométrico contribuye al conocimiento de la fundamentación teórica, el nivel de evolución y la identificación de los posibles aportes para la construcción del conocimiento [17]; por tanto, la importancia del análisis bibliométrico radica en el entendimiento más profundo del tema en cuestión y de qué manera puede hacerse un aporte teórico al campo de conocimiento.

Posteriormente, en la tercera fase, se efectuó una revisión del contexto empresarial local, donde se pusieron en evidencia los aspectos más relevantes en cuanto a desarrollo de nuevos productos, para el desarrollo de esta fase, se hizo una revisión de fuentes académicas y empresariales para determinar cómo es el proceso de desarrollo de nuevos productos en empresas locales, lo cual después de analizar datos de empresas incubadoras, organismos de apoyo y otras entidades gubernamentales, se tiene que existe un buen desempeño en cuanto a los procesos de las empresas y que se requieren de nuevas metodologías y herramientas para el desarrollo de nuevos productos con el fin de mejorar su efectividad. La importancia de esta fase radica en la comprensión del contexto de las industrias y cómo ha sido la evolución de sus procesos de desarrollo de nuevos productos para así dar paso a la propuesta de una herramienta que permita evaluar las etapas y hacer más eficiente el proceso. Al final, en la cuarta fase se elaboró la herramienta de medición enfocada al desarrollo de nuevos productos, esto fue posible gracias al contraste de la información recopilada y analizada que permitió generar, por una parte, las herramientas más adecuadas para el proceso y, por otra parte, según la información de las empresas, la construcción de una herramienta teniendo en cuenta las bases teóricas ya establecidas, por ejemplo, las etapas o puertas del State-Gate y la evaluación del Balanced Scorecard, también se consideró la adaptabilidad para las empresas objeto de estudio, las cuales son las de la industria manufacturera; por lo tanto en ésta fase se constituye por tanto en el aporte teórico y práctico del estudio.

Así mismo, es relevante mencionar que, para la realización de estas etapas, se identificaron fuentes de

Fuente: Elaboración propia.

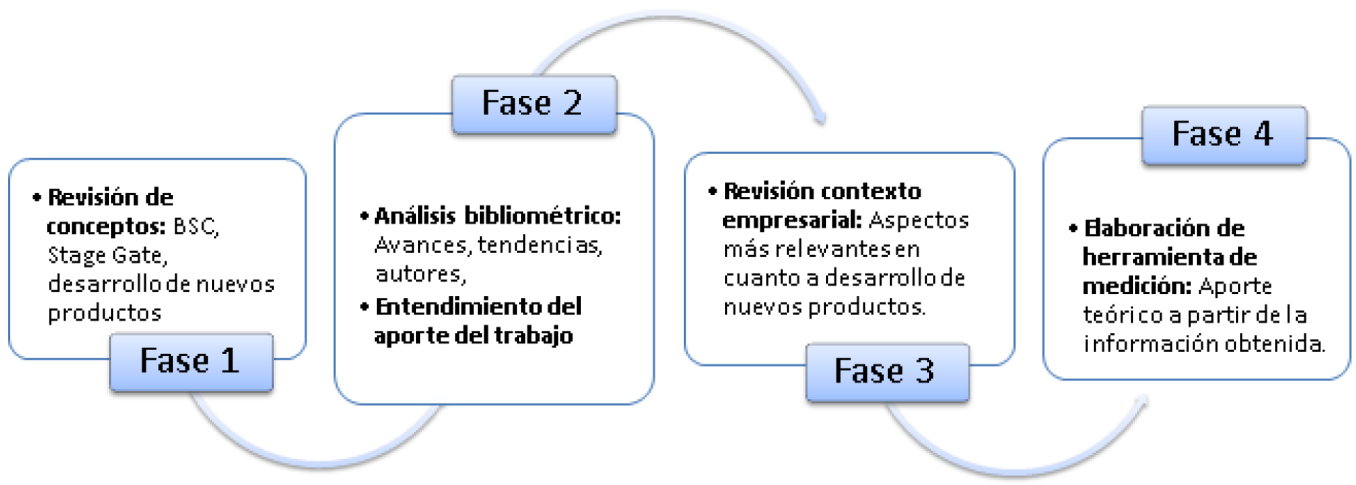

Figura 3. Fases para la construcción de la herramienta de medición y evaluación para el desarrollo de nuevos productos. 
información tales como bases de datos electrónicas entre las que se encuentran Scopus, Science Direct, Springer y Web of Science, en donde se encuentran revistas especializadas en temáticas relacionadas a los temas clave. De otro lado, fueron empleadas fuentes informales -diferentes a las revistas especializadasrelacionadas al ámbito empresarial, en las cuales fue posible encontrar casos de estudio, experiencias y otras fuentes en las que se evidencia la actualidad empresarial.

\section{RESULTADOS}

De acuerdo a la información encontrada y teniendo en cuenta el éxito que puede darse para las organizaciones tras el desarrollo de nuevos productos [18], se tomaron los modelos del Balanced Scorecard -BSC-y el State-Gate, estos se escogieron teniendo en cuenta los aportes teóricos recogidos en la literatura, fuentes humanas y fuentes empresariales, a partir de las cuales se realizó un análisis y depuración para generar la construcción de la herramienta; desde el análisis bibliométrico, se obtuvieron datos importantes en materia de tendencias y se constituyó como la fuente de referencia para entender y generar el aporte práctico de la investigación. Desde el análisis de las herramientas y el contexto empresarial, se analizaron afinidades y se extrajeron sus principales beneficios y utilidades con el fin de crear un nuevo cuadro de mando integral, construyendo una herramienta que permitiera la visualización del desarrollo de nuevos productos, definiendo objetivos estratégicos que puedan ser llevados a cabo hacia un exitoso cumplimiento.

Así, con base en cada una de las características del State-Gate y el BSC, se planteó una herramienta cualitativa, formando un nuevo método de medición de innovación en productos.

De este modo, como puede apreciarse en las siguientes figuras, se expone al lector la herramienta completa desarrollada, sin embargo, para efectos de practicidad, ésta será detallada dividiéndola en dos partes para su mayor entendimiento y capacidad de análisis. Su división se expondrá en parte 1 -el cual es el lado izquierdo- y parte 2 -el lado derecho-, tal como lo representan las Figuras 4 y 5 .

Con la unión entre ambas herramientas, se creó el Balanced Scorecard del proceso de desarrollo de nuevos productos, como una propuesta para seguir muy de cerca este aspecto, además se plantearon objetivos estratégicos para cada etapa, acompañados de indicadores que permitirán medir el estado de cada objetivo y en caso de no cumplirse, no se podrá avanzar hacia la siguiente etapa, que para el caso donde se indica Mapa Estratégico -Figura 4 parte 1-, son los mismos objetivos pero en forma de etapa y de manera secuencial, lo que provee un mayor control y orden en el proceso.

Esta herramienta plantea cinco puertas con sus respectivas actividades: i) Definición de ideas: se desarrolla una lluvia de ideas, después de su evaluación, se realiza la elección de la idea con más probabilidad de éxito, lo cual se define con investigaciones preliminares para una buena decisión; ii) Construcción del planteamiento: En esta etapa se realizan investigaciones más profundas, necesarias para determinar el mercado, presupuesto, fabricación, ventaja competitiva, entre otras, lo que dependerá del tipo de proyecto a desarrollar; iii) Desarrollo: Al definir el producto, se procede a determinar la metodología o herramienta a utilizar para la elaboración del prototipo. Esto ayudará a determinar el tiempo que se necesitará para lanzar el producto al mercado; iv) Prueba y validación: Antes de lanzar el producto se deben realizar pruebas del prototipo para analizar posibles fallas, igualmente es preciso tener en cuenta la opinión de los clientes a los que se desea llegar. Con todo definido, se puede hacer solicitud de la patente de innovación para lanzar el producto con seguridad al mercado; y v) Lanzamiento y revisión post lanzamiento: $\mathrm{Se}$ controlan los resultados y se analiza la producción, de esta manera se determina el porcentaje de participación en el mercado y concluye con un proyecto exitoso.

En esta primera parte es en donde radica la importancia de la herramienta, pues es donde se definen las puertas, que a su vez se precisan en etapas y objetivos, junto con sus indicadores, herramientas y las acciones de mejora. Esta primera parte es la gran propuesta con la que se pretende, controlar el proceso de desarrollo de nuevos productos.

Posteriormente, se cuenta con un apartado denominado Programas de acción-Figura 5 parte 2- en el cual, en caso de presentarse alguna falla o detectar una oportunidad de mejora, se pueden realizar propuestas 


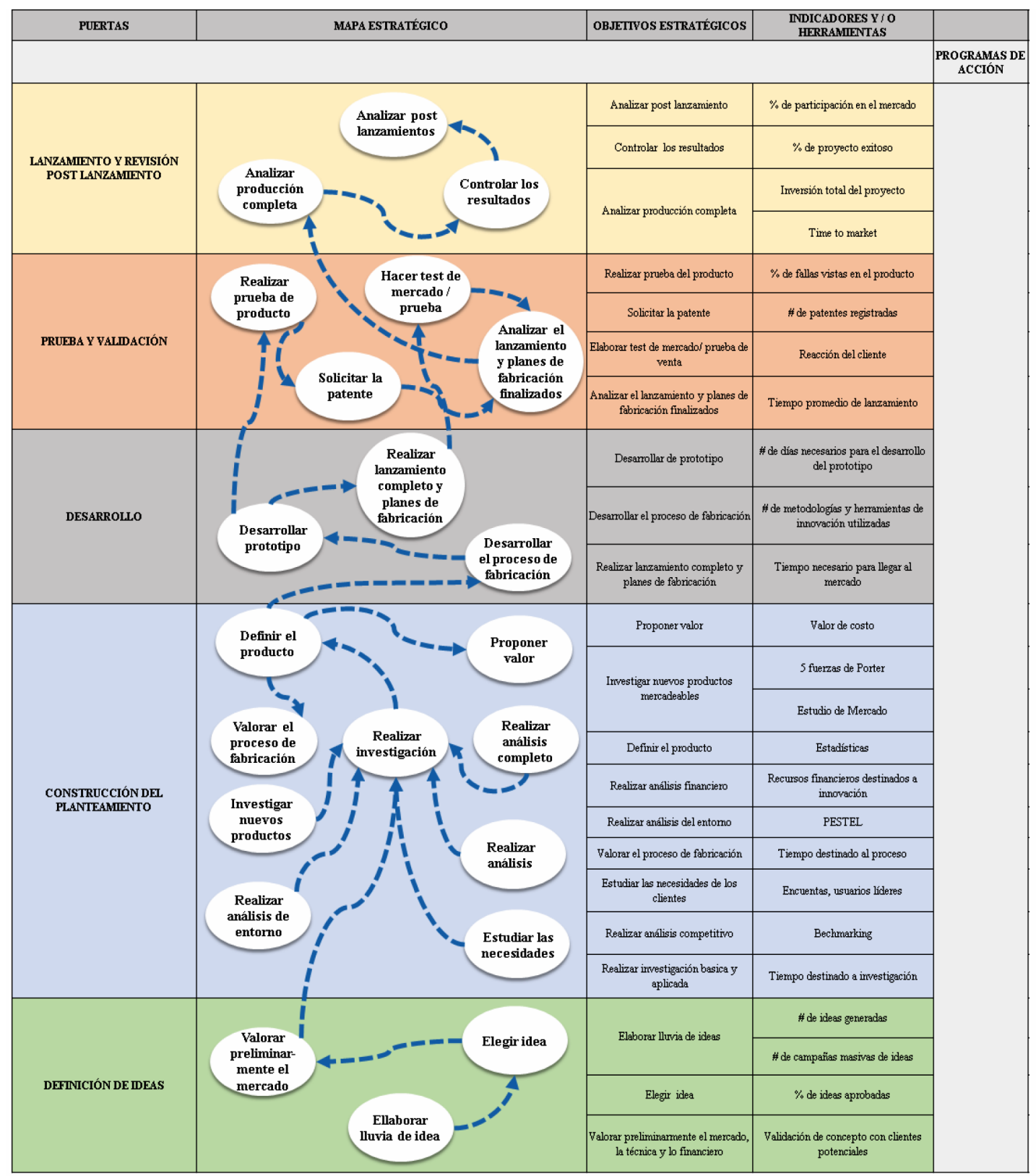

Figura 4. Herramienta de medición y control a partir del BSC y Stage - Gate: Balanced Scorecard del proceso de desarrollo de nuevos productos, parte 1: Puertas vs Objetivos y herramientas e indicadores.

para perfeccionar los objetivos o etapas completas. Una vez definido esto, se procedió a realizar la segunda fase de la herramienta denominada Mapa estratégico, el cual se compone de los objetivos estratégicos y las relaciones causales, que son la explicación de las relaciones entre los objetivos y muestran aquello que se quiere conseguir, estos mapas ayudan a valorar la importancia de cada propósito, ya que están agrupados en perspectivas que son aquellas dimensiones críticas claves [11].

Para darle un mejor control al mapa estratégico, se ha propuesto un mecanismo para evaluar constantemente cada uno de los objetivos que se van logrando 


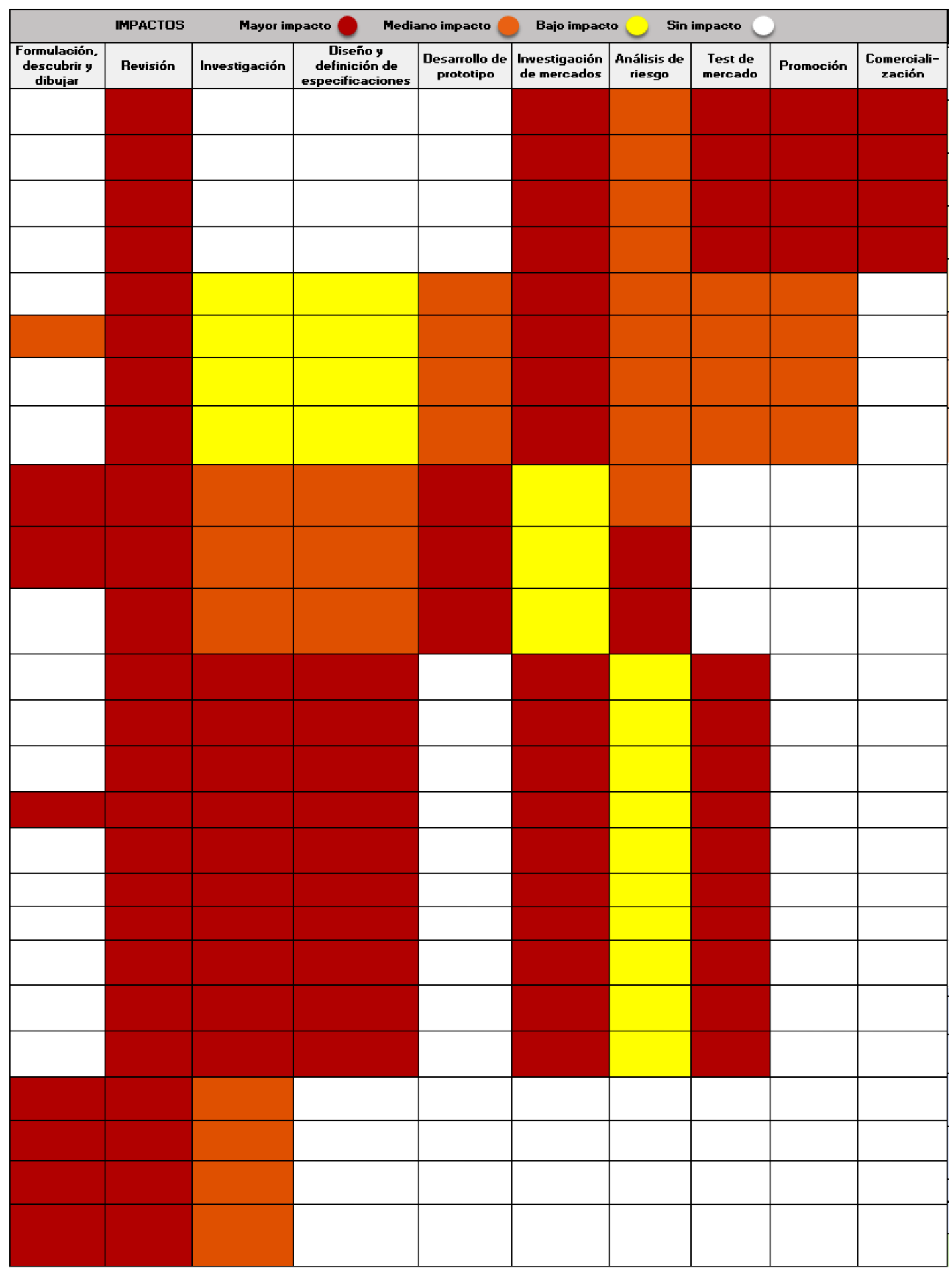

Figura 5. Herramienta de medición y control a partir del BSC y Stage-Gate: Balanced Scorecard del proceso de desarrollo de nuevos productos, parte 2: Impactos en los programas de acción vs objetivos y herramientas e indicadores. 
durante el proceso, para esto, serán marcados con tres colores de acuerdo con su estado: bien -verde-, normal -amarillo- y mal -rojo-.

Estos objetivos estratégicos dependen de unas tareas o iniciativas en las cuales se analizará el impacto que tienen y se podrá visualizar su aporte, así como el valor al cumplimiento de los objetivos. En el desarrollo de éstos, las iniciativas tienen un nivel de impacto en las etapas, por lo que cada nivel será representado por un color de la siguiente manera: rojo -mayor impacto-, verde -mediano impacto-, amarillo -bajo impacto- y blanco -sin impacto-.

La segunda parte, muestra con una escala de colores, la modelación de cómo podría ser el desarrollo de la herramienta. Si bien presenta algunos objetivos y la propuesta de medir su importancia en función de las puertas -ya que se presenta como una herramienta cruzada-, queda a consideración de las personas encargadas del proceso de desarrollo de nuevos productos su adaptación, según las condiciones particulares de cada organización; lo que hace de esta propuesta una apuesta diferencial, donde si bien se emplean fundamentos de control gerencial ampliamente explorados en la literatura internacional -como el Balanced Scorecard-, tiene el valor agregado de ser una idea emergente desde el contexto latinoamericano.

\section{DISCUSIÓN}

El desarrollo del capitalismo como modo de producción imperante en el mundo, ha planteado una serie de cambios sustanciales en la dinámica planetaria, derivando de ellos la globalización económica, lo cual ha llevado a que las sociedades actuales experimenten una serie de transformaciones en diversas dimensiones; donde las organizaciones tanto públicas como privadas siendo actores claves de la estructura económica, han tenido que adaptarse en búsqueda de la creación de ventajas competitivas que les aseguren su adaptabilidad al nuevo ritmo en que avanza el mundo [19].

Lo anterior, cobra relevancia al considerar que la globalización ha tomado mayor impulso desde mediados del siglo XX y ha llevado a aseverar el rompimiento de los límites geográficos en los países, puesto que el comercio internacional -medido por el movimiento de la balanza comercial entre importaciones y exportaciones- ha propiciado una visión sistémica del mundo, donde las dinámicas globales pueden alterar a todo el sistema, quedando en evidencia con las grandes crisis financieras y el impacto en la estabilidad financiera de los Estados -independientemente del hemisferio geográfico donde se localicen- [20]. Razón por la cual, las organizaciones y el mismo Estado nunca han estado más expuestos al riesgo, por lo que las empresas -sean públicas o privadas- precisan administrar dicho riesgo, para asegurar su sostenibilidad en el medio.

De este modo, en la medida que las sociedades se han intercomunicado más, se hace más necesario la creación de nuevos productos y servicios, que permitan a las organizaciones responder a las necesidades y deseos de las personas. Por ello, el estímulo de acciones innovadoras en las empresas con todos sus componentes científicos, financieros, comerciales y tecnológicos llevan a la creación de innovaciones al mercado; con lo cual diversos autores han hecho referencia a las ventajas que tiene para las organizaciones el fomento de la innovación, lo cual les permite obtener un mejor comportamiento económico y financiero, además de poder identificar nuevas oportunidades en el mercado [21].

Sin embargo, las organizaciones precisan no sólo la generación de productos y servicios innovadores, sino también una adecuada gestión de estos y trazabilidad frente a los procesos que intervienen en el desarrollo de innovaciones al interior de las empresas. En este sentido, el estudio aquí expuesto ha dejado una propuesta de una herramienta que permita a las compañías efectuar la medición y evaluación de este tipo de actividades, partiendo de elementos clave del Balanced Scorecard y el State-Gate.

En este sentido, la herramienta propuesta se constituye en un insumo significativo no sólo para el sector empresarial, sino también para la academia, al ser una formulación fundamentada entre insumos teóricos relevantes, pero también con un análisis riguroso del entorno al que se enfrentan las organizaciones; máxime cuando las aproximaciones realizadas por otros autores encontradas en la literatura develan el uso de otro tipo de herramientas para explorar la gestión de la innovación empresarial.

Así, Arango, Betancourt y Martínez refieren el uso del Test de Catalán para el diagnóstico de fortalezas 
y debilidades en una empresa de calzado colombiana; además de la aplicación de la herramienta CEEI ALBACETE, por la cual se hace posible la valoración de ítems como: nuevas ideas de producto, desarrollo de producto, procesos de innovación, liderazgo, asignación de recursos a la innovación, tecnología y know how [22].

Igualmente, desde el contexto colombiano se han formulado otras aproximaciones al tema, partiendo del reconocimiento frente a la complejidad existente en la gestión de proyectos en $\mathrm{I}+\mathrm{D}+\mathrm{i}$ por riesgos tecnológicos, de mercado y financieros; para ello Olaya y Duarte han propuesto una metodología para la identificación y valorización de las capacidades empresariales para el desarrollo de este tipo de proyectos; por lo cual su modelo toma como bases teóricas el Quality Function Deployment (QFD) y los Sistemas de Computación por Palabras (SCP), lo que posibilita el establecimiento de puntos críticos para este proceso [23].

Por otro lado, Salazar, Arzola y Pérez han propuesto un modelo de gestión de la innovación para las PYMIS - pequeñas y medianas industrias- en la ciudad de Guayana en Venezuela, en el cual se tomaron como referentes los Modelos de Excelencia Organizacional, así como aspectos de la Norma Española Experimental 166000 para la gestión de I+D+i, los Manuales de Bogotá y Oslo, además del Cuadro Europeo de Indicadores [24].

Desde otros contextos diferentes al escenario suramericano, en México, Aranda, De La Fuente y Becerra, han diseñado un instrumento para evaluar las prácticas de gestión de la innovación tecnológica en Pequeñas Medianas Empresas -PYME-del sector agroindustrial en el Estado de Chihuahua. Para ello tomaron como ejes referenciales el concepto de innovación dado por la Organización para la Cooperación y el Desarrollo Económico -OCDEmediante el Manual de Oslo y modelos de gestión tecnológica, calidad y productividad del ámbito mexicano [25].
Empero, pese a que se develan diferentes aproximaciones en el contexto latinoamericano para evaluar la gestión de la innovación en las organizaciones, es posible apreciar que éstas coinciden con la propuesta expuesta como resultado de este manuscrito, en que tomar fundamentos teóricos relevantes para construir nuevas herramientas, lo que les otorga rigor metodológico desde soportes de la literatura científica. Sin embargo, ninguna de ellas efectúa la hibridación planteada entre el Balanced Scorecard y el State-Gate; lo cual le confiere al estudio expuesto un aporte significativo en el sector académico y productivo latinoamericano; especialmente si se toma en cuenta el vínculo creado entre la herramienta del BSC y la competitividad de las organizaciones [26], elemento clave para el fomento de la innovación.

Finalmente, es válido destacar que en el ejercicio de discusión que se da frente a la temática abordada en el manuscrito contra las contribuciones afines o con puntos en común, no se encontraron aproximaciones coincidentes con la herramienta planteada en el contexto latinoamericano, sin embargo, si fue posible apreciar estudios en otras latitudes, como el expuesto por Al Ashaab, Flores, Doultsinou y Magyar, quienes desde el contexto de dos organizaciones - una suiza y otra británica- proponen un nuevo cuadro de mando integral -basado en el BSC de Norton y Kaplan- para evaluar el impacto de los proyectos colaborativos entre las universidades y el sector productivo; lo que hace de su propuesta una contribución significativa, al tener en cuenta que el BSC no fue concebido para medir procesos innovadores, sino para la gestión organizacional [27]. Con lo cual, es posible afirmar la utilidad de la herramienta desarrollada en este estudio y la necesidad de profundizar en este tipo de estudios.

A continuación, la Tabla 1 hace referencia a otras herramientas relacionadas con la temática del artículo; mencionando sus fortalezas, debilidades y cita bibliográfica. 
Tabla 1. Herramienta para la medición y evaluación en el desarrollo de nuevos productos.

\begin{tabular}{|c|c|c|c|c|}
\hline Herramienta & Fortalezas (usos) & $\begin{array}{l}\text { Debilidades } \\
\text { (limitaciones) }\end{array}$ & Diferenciador & Referencia \\
\hline $\begin{array}{l}\text { Implicaciones } \\
\text { de la utilización } \\
\text { de sistemas } \\
\text { BSC (Balanced } \\
\text { Scorecard) en } \\
\text { los procesos } \\
\text { de innovación } \\
\text { de producto: } \\
\text { una aplicación } \\
\text { estratégica }\end{array}$ & $\begin{array}{l}\text { Perspectiva: financiera, } \\
\text { consumidor, interna, } \\
\text { del programa, del } \\
\text { mercado, del producto } \\
\text { y de la competencia. }\end{array}$ & \begin{tabular}{|l|} 
Carece de indicadores \\
que midan factores \\
innovadores en \\
procesos desarrollados
\end{tabular} & $\begin{array}{l}\text { La herramienta tiene } \\
\text { procesos por etapas, } \\
\text { prolongando estudios } \\
\text { en determinados } \\
\text { productos y/o servicios } \\
\text { y se diferencian de la } \\
\text { propuesta en la medición } \\
\text { y control con base a } \\
\text { los indicadores del } \\
\text { producto de acuerdo al } \\
\text { enfoque y desarrollo del } \\
\text { mismo para pruebas y } \\
\text { validaciones. }\end{array}$ & (Calvo, 2007) \\
\hline $\begin{array}{l}\text { Identificación de } \\
\text { capacidades de una } \\
\text { organización para } \\
\text { el desarrollo de } \\
\text { proyectos }\end{array}$ & $\begin{array}{l}\text { Identifica aspectos } \\
\text { críticos a la } \\
\text { administración del } \\
\text { riesgo en proyectos } \\
\text { I+D+i }\end{array}$ & $\begin{array}{l}\text { La herramienta solo } \\
\text { está diseñada para } \\
\text { modelar riesgos } \\
\text { mediante números } \\
\text { difusos y sistemas } \\
\text { de computación con } \\
\text { palabras. }\end{array}$ & $\begin{array}{l}\text { La herramienta se } \\
\text { compone de cuatro } \\
\text { puestas: Investigación, } \\
\text { innovación, Desarrollo } \\
\text { tecnológico y } \\
\text { transferencia } \\
\text { tecnológica, mientras } \\
\text { que la propuesta se } \\
\text { compone de cinco } \\
\text { puertas: definición } \\
\text { de ideas, enfoque, } \\
\text { desarrollo, prueba y } \\
\text { validación. }\end{array}$ & $\begin{array}{l}\text { (Sofía \& } \\
\text { Escobar, } \\
2012 \text { ) }\end{array}$ \\
\hline $\begin{array}{l}\text { Herramientas para } \\
\text { el diagnóstico de } \\
\text { innovación en } \\
\text { una empresa del } \\
\text { sector calzado en } \\
\text { Colombia }\end{array}$ & $\begin{array}{l}\text { Permite establecer } \\
\text { un test que evalúa } \\
\text { las capacidades de } \\
\text { innovación de una } \\
\text { empresa, de acuerdo } \\
\text { a: estrategia de } \\
\text { innovación, cultura } \\
\text { de la innovación, } \\
\text { innovación en la } \\
\text { cadena de valor } \\
\text { y resultados de la } \\
\text { innovación. }\end{array}$ & \begin{tabular}{|l|} 
Los resultados \\
de aplicar esta \\
herramienta indican \\
que en la empresa, no \\
se tiene una estrategia \\
de innovación \\
completamente \\
definida por lo tanto \\
esta no puede ser \\
desplegada al máximo; \\
un aspecto que impacta \\
directamente en esto \\
es la no existencia \\
de una cultura de la \\
innovación y la falta \\
de integración de la \\
innovación a la cadena \\
de valor de la empresa.
\end{tabular} & $\begin{array}{l}\text { La herramienta } \\
\text { solo evalúa por } \\
\text { tipo de innovación } \\
\text { haciendo referencia a: } \\
\text { organización, Producto/ } \\
\text { servicio, mercado y } \\
\text { proceso mientras que la } \\
\text { propuesta del articulo } \\
\text { evalúa impactos de } \\
\text { lo que se implemente } \\
\text { de acuerdo a las } \\
\text { necesidades del cliente. }\end{array}$ & $\begin{array}{l}\text { (Alzate, } \\
\text { Hurtado, \& } \\
\text { Lopez, 2016) }\end{array}$ \\
\hline
\end{tabular}

Fuente: Elaboración propia tomando como referencia las herramientas estudiadas. 


\section{CONCLUSIONES}

El proceso de desarrollo de nuevos productos acompañado por la gestión de la innovación es fundamental para que las empresas crezcan y generen rentabilidad, además para que éstas puedan fomentar empleo y promover la competitividad del país, impulsando el desarrollo económico. Sin embargo, esto sólo es posible cuando se dispone de herramientas de planeación estratégica que posibiliten a las empresas llevar a cabo las funciones de planeación, organización, dirección y control eficazmente.

Es así como diversos autores han creado modelos que han sido de gran utilidad para las industrias, destacando entre ellos el State-Gate, empleado durante el proceso de innovación del producto desde la concepción de la idea hasta que es lanzado al mercado, dentro de éste se desarrollan actividades en cada etapa de cada puerta y están diseñadas para reunir información y disminuir progresivamente la incertidumbre, además de servir como método para transformar de forma rápida y rentable el desarrollo de las nuevas ideas en productos exitosos.

Por otro lado, se destaca el Balanced Scorecard como modelo de planeación estratégica que permite a las organizaciones tener un panorama general de su orientación en el mercado, con sus perspectivas, objetivos, relacionamiento de causa y efecto entre estos, y sus respectivos indicadores dentro de la herramienta, que muestran el camino correcto y la validación de si se está cumpliendo o no con las metas establecidas durante el proceso.

En este sentido, la herramienta diseñada mediante este estudio estuvo orientada por estos dos modelos, permitiendo hacer seguimiento y control en cada una de las etapas en el desarrollo de nuevos productos, ya que cada etapa exige planificar, organizar, definir y priorizar cada una de las actividades a realizar, y así se pueda lograr el cumplimiento de cada uno de los objetivos principales en el desarrollo global de la innovación.

Por tanto, su utilidad puede ser vista desde dos aristas: una académica y otra para el sector productivo. Frente a la primera, el desarrollo de la herramienta devela nuevos retos para investigadores y comunidad académica interesada en aportar al campo de conocimiento de la gerencia estratégica y la innovación, reconociéndoles como un binomio clave en la formación de los próximos emprendedores y empleados del mercado laboral; además de develar la versatilidad de herramientas como el BSC que usualmente se han empleado en otras áreas y no tan comúnmente en la gestión de la innovación, y la utilidad cuando se cruza con otros modelos afines al campo, en este caso el State-Gate.

Desde la segunda arista planteada, la herramienta propuesta se consolida como una aproximación que provee un insumo formulado desde el rigor de la academia y se dirige al sector productivo, que en una era globalizada demanda cada vez más la cooperación de las Instituciones de Educación Superior, para asegurar que el conocimiento generado en estas pueda ser aplicable al contexto de las organizaciones; reconocidas como actores clave para la dinamización de la economía.

Empero, pese a reconocer las bondades de la herramienta construida, no puede desconocerse que, para asegurar su utilidad y efectividad en el sector productivo, es preciso que pueda ser implementada para el fin propuesto en este estudio. Por ende, su limitación radica en ser una propuesta teórica, cuyo valor será apreciable con mayor ahínco sólo cuando pueda efectuarse un pilotaje de la herramienta y a través de resultados en el sector empresarial, valorar la contribución de ésta en las organizaciones.

\section{REFERENCIAS}

[1] S. Meeampol and A. Distanont. "Transaction cost analysis in new product development process". Advanced Sciences Letters. Vol. 23, Issue 11, pp. 10549-10554. 2017. ISSN: 1936-6612. URL: https://doi.org/10.1166/asl.2017.10100

[2] R. Lonkar and S. Gupte. "New Product Development and Innovation for Sustainable Profitable Businesses of Indian Small and Medium Scale Industries (SMEs) and Startups". Indian Journal of Public Health Research \& Development. Vol. 8, Issue 4, pp. 993-1000. 2017. ISSN - e: 0976-5506. DOI: 10.5958/0976-5506.2017.00460.0.

[3] R.G. Cooper. "Stage-gate systems: A new tool for managing new products". Business Horizons. Vol. 33, Issue 3, pp. 44-54. 1990. ISSN: 0007-6813. 
[4] R.S. Kaplan and D.P. Norton. "The Balanced Scorecards, Measures that drive performance". The Harvard Business Review. Vol. 70, Issue 1, pp. 1-19. 1992. ISSN: 00178012. D. Jiménez y R. Sanz. "Efectos de la estrategia de innovación en el éxito de los nuevos productos: el papel moderador del entorno". Revista Europea de Dirección y Economía de la Empresa. Vol. 21 No $^{\circ}$ 4, pp. 323-332. 2012. ISSN: 1019-6838. URL: https://doi. org/10.1016/j.redee.2012.07.005

[6] M. Schilling and F. Martínez. "Dirección estratégica de la innovación tecnológica". McGraw-Hill. España. 2008. ISBN: 9788448165994.

[7] E. Moones, E. Yahia and L. Roucoules. "Design process and trace modelling for design rationale capture". Joint Conference on Mechanical, Design Engineering \& Advanced Manufacturing. Tolouse, France. Jun, 2015.

[8] A. Calvo. "Implicaciones de la utlización de sistemas bsc (balanced scorecard) en los procesos de innovación de producto: una aplicación estratégica". Conocimiento, innovación y emprendedores, camino al futuro. Vol. 1, pp. 1540-1558. 2007.

[9] J. Arias. "Análisis para implementación del modelo State-Gate dentro del proceso de innovacion a nivel empresarial caso de estudio: Ezgo S.A.", pp. 7-30. 2016. URL: http://bdigital.unal.edu.co/54062/7/ JuanCarlosArias.2016.pdf

[10] H. Huang. "Designing a Knowledge -based system for strategic planning: A Balanced Scorecard Perspective". Expert Systems with Applications. Vol. 36, Issue 1, pp. 209-218. 2009. ISSN: 09574174. URL: https://doi. org/10.1016/j.eswa.2007.09.046

[11] A. Fernández. "El Balanced Scorecard". Revista de antiguos alumnos del IESE. Mar., 2001.

[12] R. Malagueño, E. Lopez-Valeiras and J. Gomez. "Balanced scorecard in SMEs: effects on innovation and financial performance". Small Business Economics. Vol. 51, Issue 1, pp. 221-244. 2017. ISSN -e: 1573-0913.

[13] A. Dávila. "Nuevas herramientas de control: el cuadro de mando integral", pp. 34-42. 1999. URL: http://planuba.orientaronline.com.ar/ wp-content/uploads/2009/10/20c-el-cuadrode-mando-integrl-antonio-davila-iese.pdf
[14] O. García. "Administración Financiera, Fundamentos y Aplicaciones". 2009.

[15] S. Scaramussa, V. Reisdorfer and A. Ribeiro. "The Contribution of the Balanced Scorecard as a Strategic Management tool in Management Support". Visión de Futuro. Vol. 13, Issue 1. 2010. ISSN -e: 1668-8708.

[16] M. Santos y E. Fidalgo. "El balanced scorecard o Cuadro de Mando Integral y el Cuadro de Mando Tradicional: principales diferencias El Balanced Scorecard (BSC)", pp. 13-17. 2005. URL: http://jggomez.eu/z\%20Privado/b\%20 usuarios/n-revista/caja/3tc/2005/673.pdf

[17] J. Aguirre. "Inteligencia estratégica: un sistema para gestionar la innovación". Estudios Gerenciales. Vol. 31 No $^{\circ} 134$, pp. 100-110. 2015. ISSN: 0123-5923. URL: https://doi. org/10.1016/j.estger.2014.07.001

[18] M. Cruz y R. Puente. “'Hay verdadera innovación en los lanzamientos de nuevos productos? Factores relevantes de éxito y fracaso en el caso colombiano". Estudios Gerenciales. Vol. 28 NN$^{\circ} 122$, pp. 263-280. 2012. ISSN: 0123-5923.

[19] C. Vargas y A. Peña. "La globalización económica y contable: Su incidencia sobre la fiscalidad en Colombia". Actualidad Contable FACES. Vol. 20 N$^{\circ}$ 35. 2017. ISSN: 1316-8533.

[20] D. Ffrench. "Globalización económica y desarrollo nacional: evolución y algunos desafíos actuales". Estudios Internacionales. Vol. 49 N Spe. 2017. ISSN -e: 0719-3769.

[21] J. Burgos y M. Lalángui. "Innovación: Tensiones teóricas en su abordaje". Revista Científica Yachana. Vol. 4, pp. 37-48. 2015. ISSN -e: 2528-8148.

[22] B.A. Alzate, J.B. Hurtado y L.F.M. Lopez. "Implementación De Herramientas Para El Diagnóstico De Innovación En La Empresa Novaflex Del Sector Calzado En Colombia". Review of Administration and Innovation RAI. Vol. 12, Issue 3, p. 310. 2016. URL: https://doi.org/10.11606/rai.v12i3.100946

[23] E. Sofía y O. Escobar. "Identificación de capacidades de una organización para el desarrollo de proyectos sensibles al riesgo mediante el despliegue de función calidad apoyado con técnicas difusas". Revista Ciencias Estratégicas. Vol. $20 \mathrm{~N}^{\circ} 28$, pp. 255 270. 2012. 
[24] M. Salazar, M. Arzola y E. Pérez. "Gestión de la innovación para las Pymes de ciudad Guayana". Revista Venezolana de Gerencia. Vol. 15 No 51, pp. 446-461. ISSN: 1315-9984.

[25] H. Aranda, M. De La Fuente y M. Becerra. "Propuesta metodológica para evaluar la gestión de la innovación tecnológica (GIT) en pequeñas y medianas empresas (PYMES)". Revista Mexicana de Agronegocios. Año 14. Vol. 26, pp. 226-238. 2010. ISSN: 2310-2799.
[26] C. Montoya. "El Balaced Scorecard como herramienta de evaluación en la gestión administrativa". Visión de Futuro. Vol. 15 $\mathrm{N}^{\circ}$ 2. 2011. ISSN: 1668-8708.

[27] A. Al -Ashaab, M. Flores, A. Doultsinou and A. Magyar. "A Balanced Scorcard for measuring the impact of industry -university collaboration". Production Planning \& Control. Vol. 22 Issue 5-6, pp. 554-570. 2011. ISSN -e: 1366-5871. URL: https:// doi.org/10.1080/09537287.2010.536626 